\title{
Age-related changes of standardized uptake values in the blood pool and liver: a decade-long retrospective study of the outcomes of 2,526 subjects
}

\author{
Yuan Cao, Ke Zhou, Wei Diao, Xipeng Long, Fangfang Tian, Minggang Su, Zhiyun Jia \\ Department of Nuclear Medicine, West China Hospital of Sichuan University, Chengdu, China \\ Correspondence to: Dr. Minggang Su; Prof. Zhiyun Jia. Department of Nuclear Medicine; Department of Nuclear Medicine, West China Hospital, \\ Sichuan University, 37 Guo Xue Alley, Chengdu 610041, China. Email: suminggang@sina.com; zhiyunjia@hotmail.com.
}

\begin{abstract}
Background: Background activity on fluorine-18-fluorodeoxyglucose $\left({ }^{18} \mathrm{~F}\right.$-FDG) positron emission tomography/computed tomography (PET/CT) is often used as a reference to assess a patient's response to tumor treatment. To produce a suitable background activity reference, we examined the variations in standardized uptake values (SUVs) in the blood pool and liver of a large multi-aged population.

Methods: A total of 2,526 subjects underwent ${ }^{18} \mathrm{~F}$-FDG PET/CT examinations and were divided into 12 age groups. Pearson's partial correlation and multivariate regression analyses were performed to assess the associations between individual factors and SUVs of the blood pool and liver and to identify the factor that most influenced the SUVs. The mean SUVs across the age groups were also determined.

Results: Positive correlations were found between individual factors and SUVs. Age appeared to be the most important predictor of SUVs and was significantly associated with the blood pool $\mathrm{SUV}_{\max }(B=0.466$, $\mathrm{P}=0.000)$, blood pool $\mathrm{SUV}_{\text {mean }}\left(\beta_{3}=0.393, \mathrm{P}=0.000\right)$, liver $\mathrm{SUV}_{\max }\left(\beta_{1}=0.347, \mathrm{P}=0.000\right)$, and liver $\mathrm{SUV}_{\text {mean }}$ $(ß=0.354, \mathrm{P}=0.000)$. Blood pool and liver SUVs rose rapidly until the age of 20 and then showed a slow upward trend without reaching a plateau.

Conclusions: Age is an important factor that influences variations in the blood pool and liver SUVs. Our study clarified this understanding of age-related variations in SUVs and provided a normal range of blood pool and liver SUVs that may aid clinicians in evaluating tumors with greater accuracy.
\end{abstract}

Keywords: Fluorine-18-fluorodeoxyglucose positron emission tomography/computed tomography $\left({ }^{18} \mathrm{~F}-\mathrm{FDG}-\right.$ PET/CT); standardized uptake value (SUV); liver; blood pool; age variation

Submitted Jan 07, 2020. Accepted for publication Jun 22, 2020.

doi: 10.21037/qims-20-35

View this article at: http://dx.doi.org/10.21037/qims-20-35

\section{Introduction}

Fluorine-18-fluorodeoxyglucose $\left({ }^{18} \mathrm{~F}-\mathrm{FDG}\right)$ integrated positron emission tomography and computed tomography (PET/CT) is an imaging technique that provides both anatomical and glucometabolic information that guides clinicians in distinguishing benign disease from malignant disease, as well as assessing patient response to cancer treatment $(1,2)$. Standardized uptake values (SUVs) are a useful quality index of FDG PET/CT studies at the PET/ CT image interpretation stage (3). A five-point scale (5-PS) model can be used with liver and mediastinal blood pool SUV measurements to differentiate abnormal FDG uptake from physiological FDG uptake. These measurements are often used as reference background values to distinguish tumors and define treatment response for head and neck squamous cell carcinoma and lymphoma (3-6). However, a variety of biological factors can cause errors in liver and blood pool SUVs (7). These factors may give rise to falsepositive or false-negative PET/CT reports (8) and include age, sex, weight, serum glucose level, hepatic function, and 
hyperthyroidism (6,9-11). Therefore, normal SUVs of the liver and blood pool must be determined before clinicians can interpret PET/CT images. Growing evidence suggests that age has a major impact on SUVs $(1,12)$. However, no studies have focused on variations in SUVs across different ages, possibly because of the limitations of small sample sizes. Therefore, to ensure scientific rigor and enable reproducibility, defining a precise range of blood pool and liver SUVs from ${ }^{18} \mathrm{~F}$-FDG PET/CT in multi-aged populations is of great importance.

Although a previous study of pediatric patients and adults has explored the impact of age on SUVs (1), it only provided potential associations; the relationship between different ages and the SUVs of blood pool and liver have not been fully explored. To date, there is no clear consensus on the real impact that age has on ${ }^{18} \mathrm{~F}$-FDG uptake. In addition, the mechanisms related to this association have not been determined. In this study, the mean SUVs $\left(\mathrm{SUV}_{\text {mean }}\right)$ and maximum SUVs $\left(\mathrm{SUV}_{\text {max }}\right)$ were both applied as indices of ${ }^{18} \mathrm{~F}$-FDG uptake in the blood pool and liver; the blood pool is a less variable and more robust parameter, while the liver is the most common clinical parameter for illustrating ${ }^{18} \mathrm{~F}$-FDG accumulation in tumors. We enrolled a cohort of Chinese patients, stratified them into 12 age groups for evaluation, and quantified a normal range of background blood pool and liver SUVs to guide clinicians in more accurately identifying tumors and evaluating cancer treatment response. We present the following article in accordance with the STROBE Statement guidelines. A completed STROBE reporting checklist is available at http://dx.doi.org/10.21037/qims-20-35.

\section{Methods}

\section{Selection of participants}

The research procedures in this study were approved by the West China Hospital of Sichuan University Ethics Committee. This is a retrospective study, so no ethical approval or informed consent was obtained. This retrospective study initially included all subjects who underwent ${ }^{18} \mathrm{~F}$-FDG PET/CT examinations at our institution between January 2009 and January 2019. The inclusion and exclusion criteria included the following. Imaging was performed from the top of the skull to mid-thigh, and PET was performed from the pelvis to the head with the arms elevated and a tracer uptake period of 50-75 minutes. Since this study included subjects that ranged from infant to adult to elderly, there were diverse incubation times. However, the previous study that investigated the impact of age on SUVs determined an incubation time that was within only $5 \%$ of the peak value, between 50 and $110 \mathrm{~min}$ after the injection (13). Subjects with a fever, diabetes, hematologic disease, abnormal liver or renal function, and primary or secondary hepatic and/or aortic diseases (neoplasms, large-sized cysts, aneurysms, inflammation, viral hepatitis B or $\mathrm{C}$, and hepatic adipose infiltration) were excluded from this study. Subjects with FDG-avid tumors or who had received chemotherapy within eight weeks of imaging, radiotherapy for the liver or mediastinum, or bone marrow colony-stimulating factor treatment within two weeks of imaging were also excluded. The flow diagram of subject selection is shown in Figure 1. The following data were recorded: age, sex, weight, ${ }^{18}$ F-FDG dose, serum glucose level, and the results of liver function tests. All participant data were collected by two clinicians (YC and $\mathrm{KZ}$ ) to minimize errors during the data collection process.

\section{Imaging technique}

All subjects fasted for at least 6 hours prior to the examination to maintain low glucose and low insulin levels. The ${ }^{18} \mathrm{~F}$-FDG PET/CT images were acquired after intravenous injection of $5.55 \mathrm{MBq}(0.15 \mathrm{mCi})$ of ${ }^{18} \mathrm{~F}-\mathrm{FDG}$ per kilogram of bodyweight via the cubital vein. The scanning parameters were $4 \mathrm{~mm} /$ slice for PET and $120 \mathrm{kV}$, $40 \mathrm{mAs}$, and $5 \mathrm{~mm} / \mathrm{slice}$ for low-dose CT. The emission PET images were acquired in $3 \mathrm{D}$ mode at 2 minutes per bed position and a bed overlap of $50 \%$. The reconstruction of PET images was performed according to the European Association of Nuclear Medicine Guidelines for tumor PET imaging (6) and used a line-of-response row-action maximum likelihood algorithm (3 iterations and 33 subsets, voxel size of $4 \mathrm{~mm} \times 4 \mathrm{~mm} \times 4 \mathrm{~mm}$, with no additional Gaussian smoothing). Trans-axial, coronal, and sagittal $\mathrm{CT}$ and PET/CT fusion images were reconstructed and formatted using vendor-provided software (Phillips EBW Workstation). All scans were acquired using a Gemini GXL PET/CT scanner (Philips, Netherlands).

\section{Imaging protocols and analysis}

FDG uptake was represented by SUVs, which were calculated according to the following formula (1):

$\mathrm{SUV}_{\text {max }}=$ maximum activity in region of interest (ROI) $(\mathrm{kBq}) /$ injected dose $(\mathrm{MBq}) \times$ body weight $(\mathrm{kg})$ 


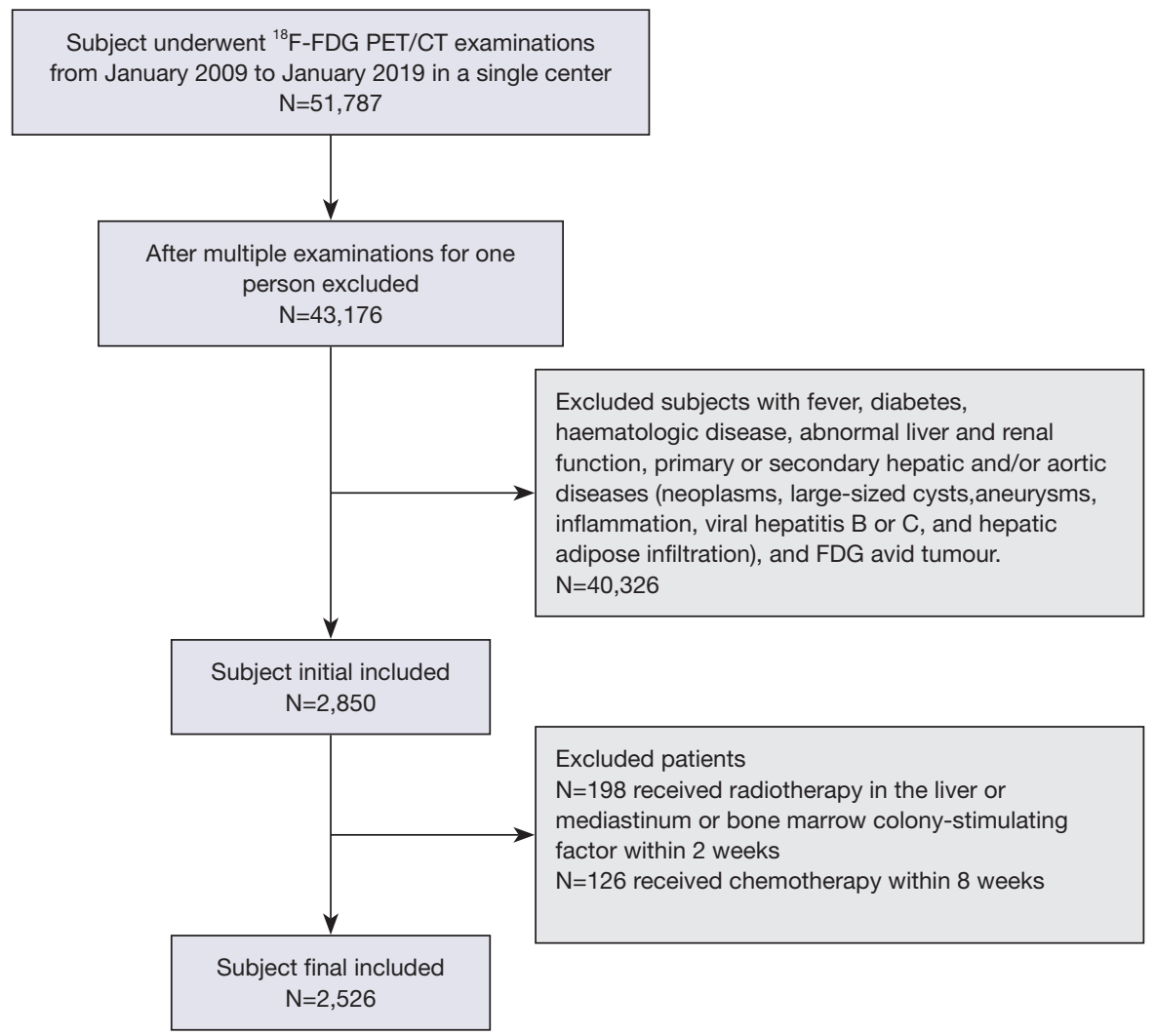

Figure 1 Flow diagram of subject selection.

$\mathrm{SUV}_{\text {mean }}=$ mean activity in ROI $(\mathrm{kBq}) /$ injected dose $(\mathrm{MBq}) \times$ body weight $(\mathrm{kg})$

A quantitative method was performed by placing a spherical volume of interest (VOI) with a diameter of $3 \mathrm{~cm}$ in the center of the right lobe of the liver while avoiding visible vessels on the CT (6) (Figure 2A,B). The mediastinal blood pool measurements were performed by drawing a combined VOI on three contiguous slices inside the thoracic aorta at the carinal level and measuring uptake within the vessel while avoiding the vessel wall $(14,15)$ (Figure $2 C, D$ ). The $\mathrm{SUV}_{\text {mean }}$ and $\mathrm{SUV}_{\text {max }}$ of the blood pool and liver were recorded (16).

\section{Statistical analysis}

All analyzes were performed using SPSS software version 22.0 (IBM Corporation, Armonk, NY, USA). Continuous variables were presented as mean \pm standard deviation $(\mathrm{SD})$. Data normality was evaluated by the Kolmogorov-Smirnov test. Pearson's partial correlation coefficients were used to evaluate the relationships of $S \mathrm{SV}_{\text {max }}$ and $S \mathrm{SUV}_{\text {mean }}$ in the blood pool and liver with age, weight, ${ }^{18} \mathrm{~F}-\mathrm{FDG}$ dose, and serum glucose level. A multivariate linear regression model was established to determine the best predictors of liver and blood pool $\mathrm{SUV}_{\text {max }}$ and $\mathrm{SUV}_{\text {mean }} . \mathrm{P}<0.05$ was considered statistically significant.

\section{Results}

Applying exclusion criteria, a total of 2,526 subjects, 1,436 males and 1,090 females, were recruited in this study. The mean $\mathrm{SUV}_{\text {max }}$ and $\mathrm{SUV}_{\text {mean }}$ in the blood pool were $1.89 \pm 0.36$ and $1.55 \pm 0.28$, respectively, while the mean $S_{U V} V_{\max }$ and $S_{U V}$ mean in the liver were $2.81 \pm 0.46$ and $2.26 \pm 0.38$, respectively. The clinical characteristics of the study populations are shown in Table 1.

\section{Pearson's partial correlation analysis}

A scatter plot matrix shows the correlations between individual factors and SUVs (Figure 3). The blood pool $\mathrm{SUV}_{\max }$ and $\mathrm{SUV}_{\text {mean }}$ had statistically significant positive 

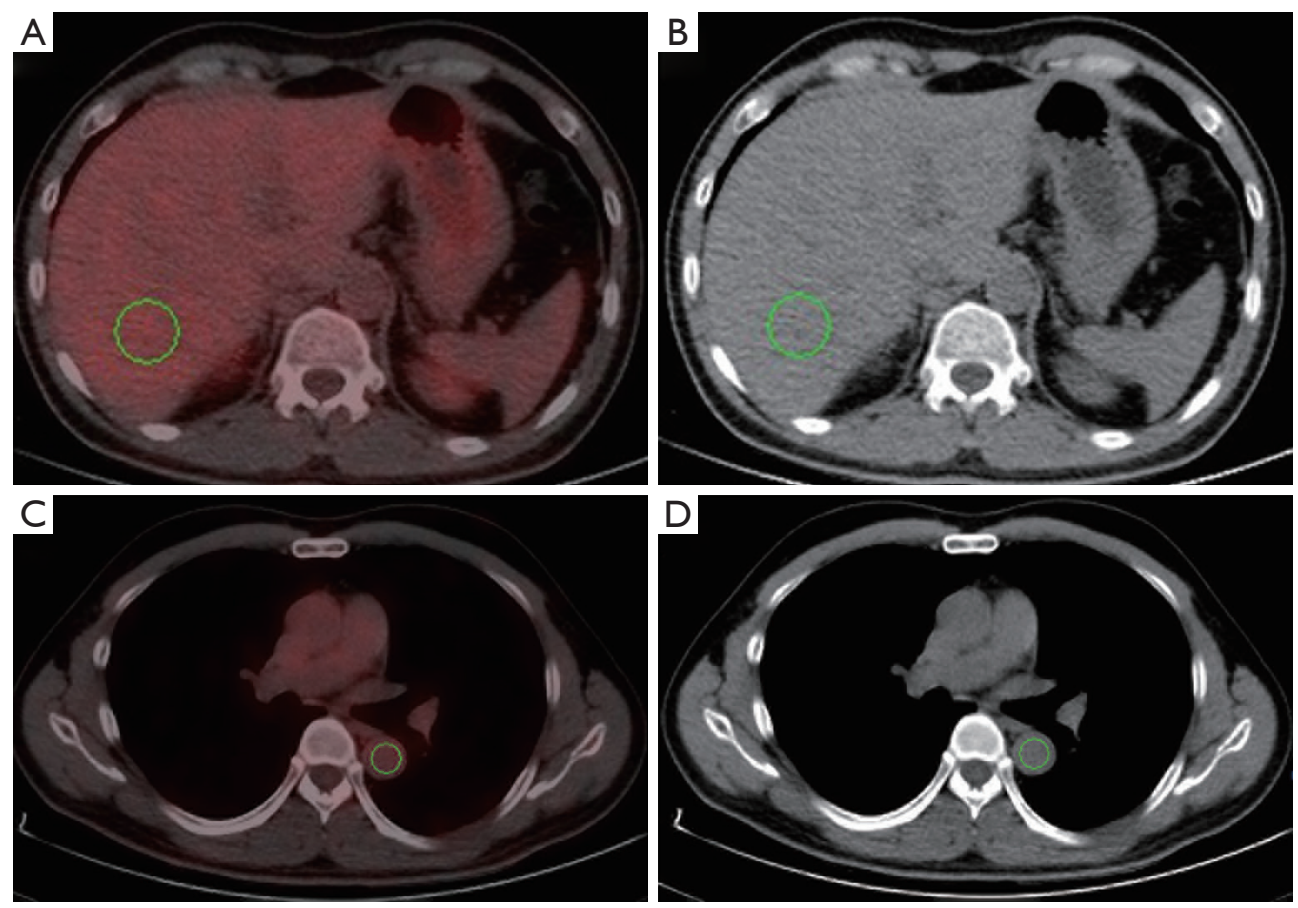

Figure 2 Images of a 40-year-old man with no cancer history. PET/CT was performed for physical examination. (A) The fusion image showed an ROI with a diameter of $3 \mathrm{~cm}$ in the center of the right lobe of the liver. (B) The CT images showed a liver of normal appearance with no sign of abnormalities. (C) The fusion image showed an ROI inside the thoracic aorta at the carinal level. (D) The CT images showed a blood pool of normal appearance with no sign of abnormalities. PET/CT, positron emission tomography/computed tomography; ROI, region of interest.

correlations with age, weight, ${ }^{18} \mathrm{~F}-\mathrm{FDG}$ dose, and serum glucose level. Of these, age had the strongest correlation with both $\mathrm{SUV}_{\text {max }}(\mathrm{r}=0.466, \mathrm{P}=0.000)$ and $\mathrm{SUV}_{\text {mean }}(\mathrm{r}=0.393$, $\mathrm{P}=0.000)$. The liver $\mathrm{SUV}_{\max }$ and $\mathrm{SUV}_{\text {mean }}$ had similar correlations with the abovementioned factors, except serum glucose level $\left(\mathrm{r}=0.017, \mathrm{P}=0.404\right.$ for liver $\mathrm{SUV}_{\max } ; \mathrm{r}=0.03$, $\mathrm{P}=0.136$ for liver $\mathrm{SUV}_{\text {mean }}$ ). Of these, age also had the strongest positive correlation with liver $\mathrm{SUV}_{\text {mean }}(\mathrm{r}=0.347$, $\mathrm{P}=0.000)$ and liver $\mathrm{SUV}_{\text {max }}(\mathrm{r}=0.354, \mathrm{P}=0.000)$ (Table 2).

\section{Multivariate linear regression analysis}

The multivariate regression analysis suggested that, of the four factors, age had the greatest impact on $\mathrm{SUV}_{\max }$ and $\mathrm{SUV}_{\text {mean }}$ in the blood pool $\left(\beta=0.462, \mathrm{P}=0.000\right.$ for $\mathrm{SUV}_{\text {max }}$; $\beta=0.385, P=0.000$ for $\left.S U V_{\text {mean }}\right)$ and in the liver $(\beta=0.324$, $\mathrm{P}=0.000$ for $\mathrm{SUV}_{\max } ; \beta=0.376, \mathrm{P}=0.000$ for $\mathrm{SUV}_{\text {mean }}$ ) (Table 3). We grouped the subjects into 12 age groups (1-5, 6-10, 11-15, 16-20, 21-25, 26-30, 31-40, 41-50, 51-60, $61-70,71-80$, and $81-100$ years). Overall, the blood pool $\mathrm{SUV}_{\text {max }}$ (from $1.06 \pm 0.24$ to $1.62 \pm 0.23$ ) and $\mathrm{SUV}_{\text {mean }}$ (from
$0.89 \pm 0.18$ to $1.35 \pm 0.24)$ increased rapidly until the age of 20. Then the growth trend continued more slowly with age, and no plateau was reached. The liver $\mathrm{SUV}_{\max }$ (from $1.38 \pm 0.25$ to $2.60 \pm 0.36$ ) and $\mathrm{SUV}_{\text {mean }}$ (from $1.10 \pm 0.20$ to $2.08 \pm 0.28$ ) showed similar patterns (Table 4, Figure 4). Similar changes were also found in PET/CT images showing maximum intensity projections (MIPs) of the blood pool and liver at different ages $(5,10,15,20,25,30,40,50$, 60, 70, 80, and 90 years) (Figure 5).

\section{Subgroup analysis}

According to the above trend, children and adolescents showed more significant growth relationships between age and SUVs. To avoid the influence of a wide age range on the results, particularly the influence of children and adolescents, which may reduce the robustness of the results, we also obtained Pearson's partial correlations and performed multivariate linear regression analysis between individual factors and SUVs in subjects older than 18 years. Similar correlations and trends were obtained in 
Table 1 Demographic data of subjects

\begin{tabular}{|c|c|}
\hline Characteristics & $\begin{array}{l}\text { Number of subjects } \\
\qquad(\mathrm{N}=2,526)\end{array}$ \\
\hline \multicolumn{2}{|l|}{ Gender } \\
\hline Male & 1,436 \\
\hline Female & 1,090 \\
\hline \multicolumn{2}{|l|}{ Category } \\
\hline Cancer screening & 1,720 \\
\hline Lymphoma & 341 \\
\hline Lung cancer & 154 \\
\hline HNSCC \& NPC & 110 \\
\hline Colorectal cancer & 102 \\
\hline Melanoma & 69 \\
\hline Ewing sarcoma & 30 \\
\hline \multicolumn{2}{|l|}{ Liver function test* } \\
\hline Alanine transaminase (serum IU/L) & $19.30 \pm 9.36$ \\
\hline Aspartate transaminase (serum IU/L) & $22.60 \pm 6.07$ \\
\hline Bilirubin $(\mu \mathrm{mol} / \mathrm{L})$ & $11.94 \pm 5.18$ \\
\hline Albumin (g/L) & $46.52 \pm 3.30$ \\
\hline Weight (kg) & $60.87 \pm 14.00$ \\
\hline${ }^{18} \mathrm{~F}-\mathrm{FDG}$ dose $(\mathrm{mCi})$ & $9.50 \pm 2.08$ \\
\hline Blood glucose (mmol/L) & $5.36 \pm 0.62$ \\
\hline Blood pool SUV $\max$ & $1.89 \pm 0.36$ \\
\hline Blood pool SUV mean & $1.55 \pm 0.28$ \\
\hline Liver SUV max $_{\text {m }}$ & $2.81 \pm 0.46$ \\
\hline Liver SUV $_{\text {mean }}$ & $2.26 \pm 0.38$ \\
\hline
\end{tabular}

*The reference range: alanine transaminase ( $<50 \mathrm{IU} / \mathrm{L})$; aspartate transaminase (<40 IU/L); bilirubin (5-28 $\mu \mathrm{mol} / \mathrm{L})$; albumin (40$55 \mathrm{~g} / \mathrm{L})$. HNSCC, head, and neck squamous cell carcinoma; NPC, nasopharyngeal carcinoma; ${ }^{18}$ F-FDG, fluorine-18fluorodeoxyglucose; $\mathrm{SUV}_{\max }$, maximum standardized uptake value; SUV mean, mean standardized uptake value; N, number.

the subgroup analysis, which validated the original results (Tables S1,S2).

\section{Discussion}

Treatment response in patients with head and neck squamous cell carcinoma and lymphoma can be assessed using 5-PS scores; this requires the comparison of SUVs of the ${ }^{18} \mathrm{~F}$-FDG-positive lesion with that of the mediastinum blood pool and liver (17). Determining a reference range of SUVs using ${ }^{18}$ F-FDG PET/CT in multi-aged populations is of great clinical utility, as previous studies have found an association between age and SUVs (1). This study further demonstrated that SUVs of the blood pool and liver were affected by age, even after adjusting for weight, injected a dose of ${ }^{18} \mathrm{~F}-\mathrm{FDG}$, and serum glucose level. Based on this 10 -year retrospective study of 2,526 patients, we found that the $\mathrm{SUV}_{\text {max }}$ and $\mathrm{SUV}_{\text {mean }}$ of the blood pool and liver increased rapidly until the age of 20 . The growth trend then slowed without reaching a plateau. More importantly, this study provided a normal range of background SUVs across 12 age groups. This could assist clinicians in selecting appropriate reference background SUVs when treating oncologic patients of different ages.

Several studies have documented the effects of age, weight, BMI, and ${ }^{18} \mathrm{~F}$-FDG dose on ${ }^{18} \mathrm{~F}$-FDG uptake in the blood pool and liver $(5,18-20)$. A study by Mahmud et al. (12) investigated these effects in 51 oncology patients, including 28 males and 23 females. In contrast to our results, no significant positive association was found between liver $\mathrm{SUV}_{\max }$ and age. This may be due to the small sample size, the inclusion of elderly patients, and $\mathrm{SUV}_{\max }$ variations. Notably, the lack of a significant correlation between serum glucose level and $\mathrm{SUV}_{\text {max }}$ was consistent with our results. Lin et al. (1) investigated the relationships of liver SUVs with sex, age, and HBV and HCV infection status in 339 asymptomatic subjects. The authors reported that age had a significant positive relationship with liver SUVs. No significant associations were observed between the other factors and SUVs. In our study, although no significant correlation was found between liver SUVs and serum glucose levels, we did find weak positive correlations between weight, ${ }^{18}$ F-FDG dose, serum glucose level, and the other SUV measurements. Moderate positive correlations between age and the blood pool and liver FDG uptake were found. Even after adjusting for confounding variables, the blood pool and liver SUVs retained a significant correlation with age. To the best of our knowledge, apart from the influence of hyperthyroidism on the liver itself $(6,9)$, there are several potential biological explanations for the agerelated trends of SUVs observed in our study. We speculate that the relevant mechanisms may be explained by organ deterioration, organ metabolism, and molecular transport.

On a cellular level, although the volume of hepatocytes grows with age, functional hepatocyte volume, and hepatocyte numbers decrease significantly with age $(21,22)$. 

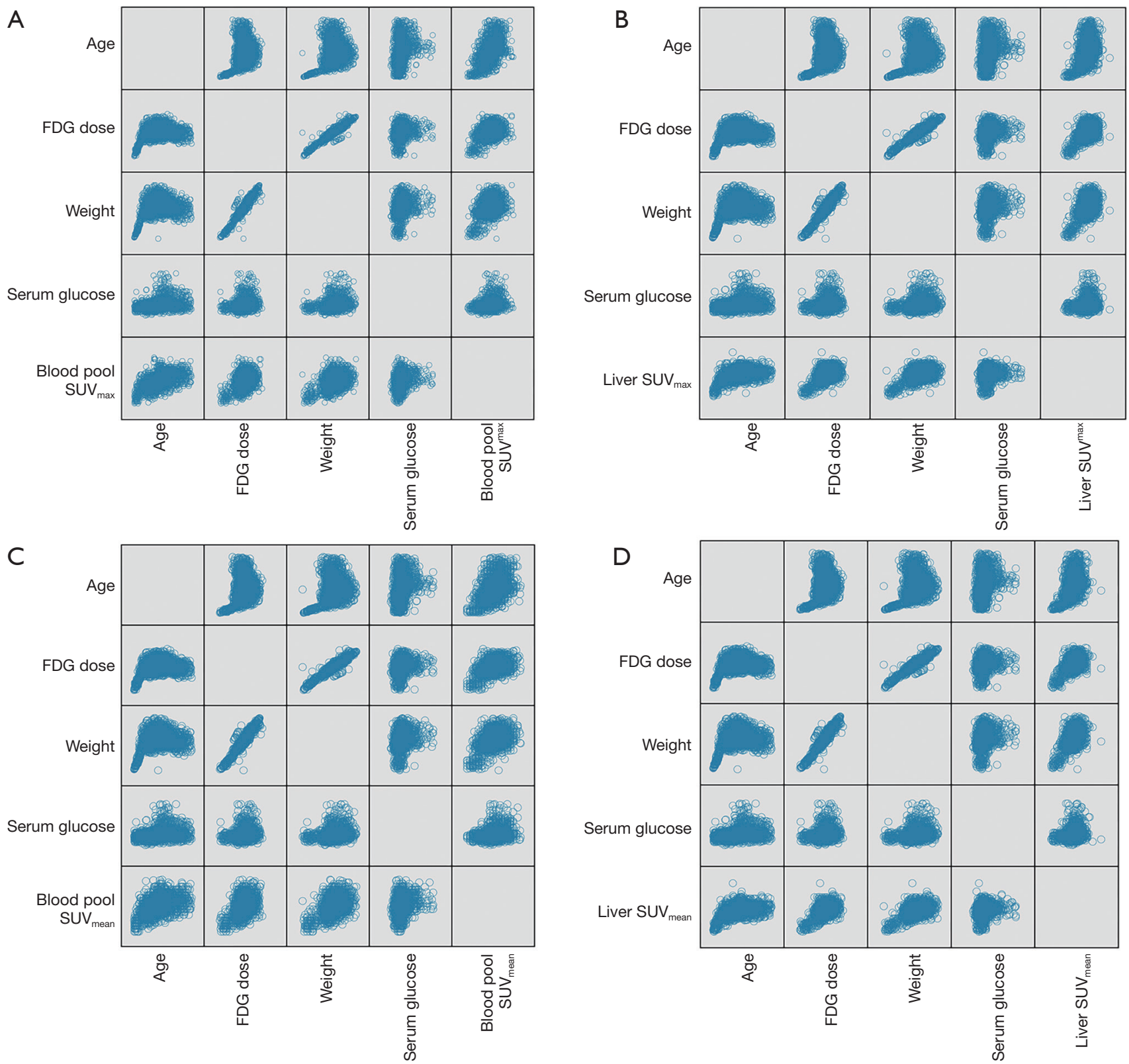

Figure 3 The correlation between individual factors and SUVs. (A) A scatter plot matrix showing the relationships between different factors and blood pool SUV max (B) A scatter plot matrix showing the relationships between different factors and liver $\mathrm{SUV}_{\max }$ (C) A scatter plot matrix showing the relationships between different factors and blood pool $\mathrm{SUV}_{\text {mean }}$ (D). A scatter plot matrix showing the relationships between different factors and liver $\mathrm{SUV}_{\text {mean }}$. SUV, standardized uptake value.

Additionally, there is a positive association between metabolic activity in the liver and age in adults (23). The increase of FDG uptake with age may also reflect agerelated metabolic activity and changes in liver volume and hepatocyte numbers, as noted by previous studies $(23,24)$. However, Meier and colleagues suggested that cumulative inflammatory changes secondary to the release of agerelated hepatotoxins may exist (23). The age-related metabolic level of the organ may be one explanation for blood pool SUV trends (25). Overall, the similarities between previous research and our study indicate that our study results are of high scientific integrity. 
Table 2 Correlations between SUVs and related factors

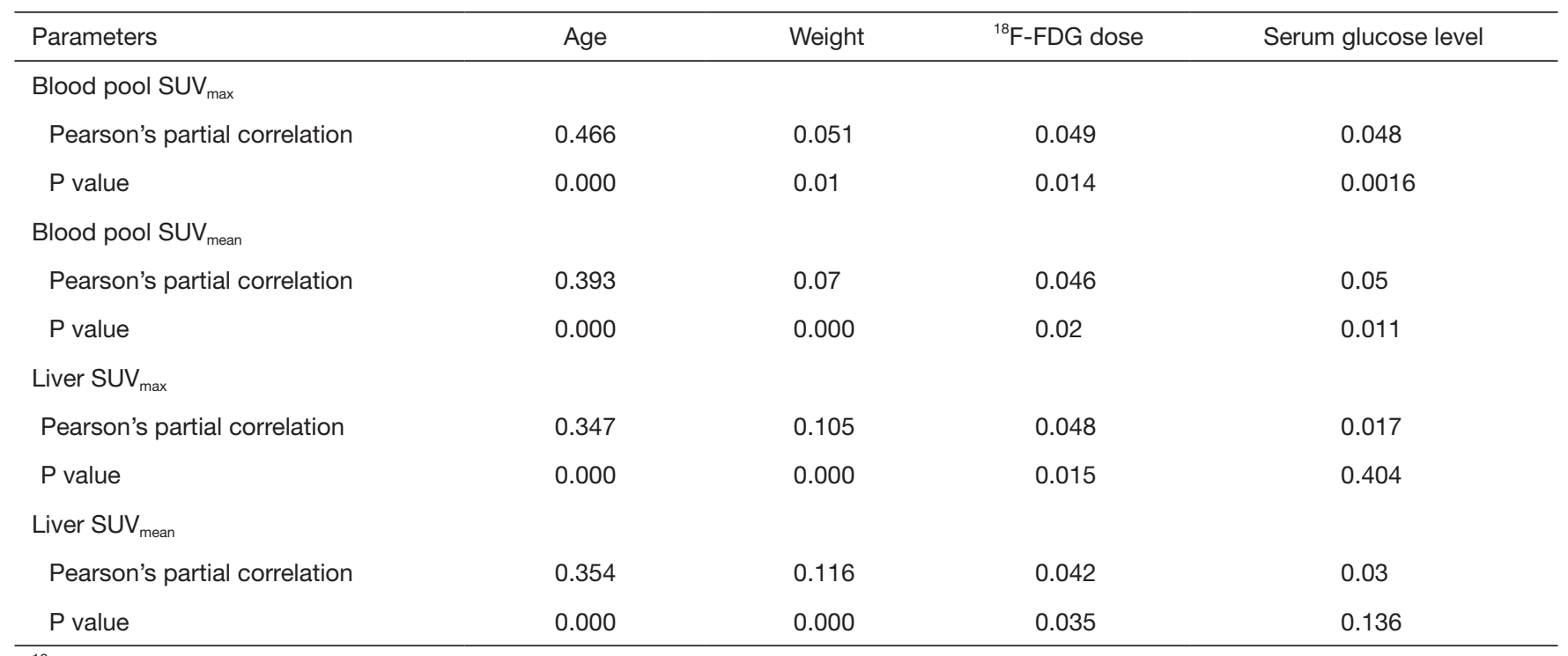

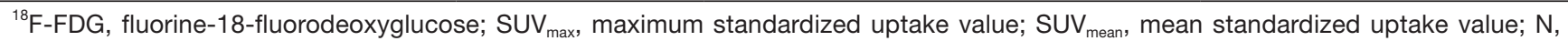
number.

Table 3 Impact of related factors on SUVs of blood pool and liver in multivariate regression analysis

\begin{tabular}{|c|c|c|c|}
\hline Variable & Adjusted unstandardized beta coefficient & Adjusted standardized beta coefficient & $P$ value \\
\hline \multicolumn{4}{|l|}{ Blood pool SUV $\max$} \\
\hline Age & 0.009 & 0.462 & 0.000 \\
\hline${ }^{18}$ F-FDG dose & 0.020 & 0.119 & 0.028 \\
\hline Weight & 0.004 & 0.164 & 0.003 \\
\hline \multicolumn{4}{|l|}{ Blood pool SUV mean $_{\text {n }}$} \\
\hline Age & 0.006 & 0.385 & 0.000 \\
\hline${ }^{18} \mathrm{~F}-\mathrm{FDG}$ dose & 0.017 & 0.125 & 0.024 \\
\hline Weight & 0.004 & 0.220 & 0.000 \\
\hline${ }^{18} \mathrm{~F}-\mathrm{FDG}$ dose & 0.030 & 0.134 & 0.013 \\
\hline Weight & 0.010 & 0.314 & 0.000 \\
\hline Serum glucose level & 0.008 & 0.015 & 0.375 \\
\hline \multicolumn{4}{|l|}{ Liver SUV $_{\text {mean }}$} \\
\hline Age & 0.007 & 0.376 & 0.000 \\
\hline${ }^{18} \mathrm{~F}$-FDG dose & 0.004 & 0.022 & 0.701 \\
\hline Weight & 0.008 & 0.284 & 0.000 \\
\hline
\end{tabular}

\footnotetext{
${ }^{18}$ F-FDG, fluorine-18-fluorodeoxyglucose; SUV $_{\max }$, maximum standardized uptake value; SUV $_{\text {mean }}$, mean standardized uptake value.
} 
Table 4 Mean SUVs of blood pool and liver in different subject age groups

\begin{tabular}{|c|c|c|c|c|c|c|c|c|}
\hline Age $[\mathrm{N}]$ & \multicolumn{2}{|c|}{ Blood pool SUV $\max$} & \multicolumn{2}{|c|}{ Blood pool SUV mean } & \multicolumn{2}{|c|}{ Liver SUV $\max$} & \multicolumn{2}{|c|}{ Liver SUV $_{\text {mean }}$} \\
\hline $1-5[47]$ & $1.06 \pm 0.24$ & $0.99-1.13$ & $0.89 \pm 0.18$ & $0.83-0.94$ & $1.38 \pm 0.25$ & $1.30-1.45$ & $1.10 \pm 0.20$ & $1.04-1.16$ \\
\hline $6-10[53]$ & $1.29 \pm 0.33$ & $1.20-1.39$ & $1.05 \pm 0.27$ & $0.97-1.13$ & $1.80 \pm 0.40$ & $1.69-1.91$ & $1.44 \pm 0.30$ & $1.36-1.52$ \\
\hline $11-15[56]$ & $1.54 \pm 0.27$ & $1.47-1.61$ & $1.28 \pm 0.25$ & $1.21-1.34$ & $2.40 \pm 0.42$ & $2.29-2.51$ & $1.88 \pm 0.34$ & $1.79-1.97$ \\
\hline $21-25$ [165] & $1.70 \pm 0.31$ & $1.65-1.74$ & $1.44 \pm 0.26$ & $1.40-1.48$ & $2.73 \pm 0.42$ & $2.67-2.80$ & $2.18 \pm 0.34$ & $2.13-2.24$ \\
\hline $26-30$ [139] & $1.67 \pm 0.28$ & $1.63-1.72$ & $1.44 \pm 0.23$ & $1.39-1.47$ & $2.72 \pm 0.35$ & $2.66-2.78$ & $2.19 \pm 0.27$ & $2.14-2.24$ \\
\hline $31-40$ [491] & $1.81 \pm 0.31$ & $1.78-1.84$ & $1.53 \pm 0.24$ & $1.50-1.55$ & $2.78 \pm 0.37$ & $2.75-2.82$ & $2.25 \pm 0.30$ & $2.22-2.28$ \\
\hline $41-50$ [638] & $1.99 \pm 0.30$ & $1.97-2.01$ & $1.62 \pm 0.24$ & $1.60-1.64$ & $2.94 \pm 0.37$ & $2.91-2.96$ & $2.35 \pm 0.30$ & $2.33-2.38$ \\
\hline $71-80$ [127] & $2.10 \pm 0.29$ & $2.05-2.15$ & $1.68 \pm 0.24$ & $1.64-1.72$ & $2.97 \pm 0.36$ & $2.91-3.03$ & $2.38 \pm 0.30$ & $2.33-2.43$ \\
\hline $81-100$ [123] & $2.17 \pm 0.29$ & $2.11-2.22$ & $1.74 \pm 0.27$ & $1.69-1.79$ & $3.01 \pm 0.36$ & $2.95-3.07$ & $2.44 \pm 0.32$ & $2.38-2.50$ \\
\hline
\end{tabular}

$\mathrm{SD}$, standard deviation; $\mathrm{Cl}$, confidence interval; $\mathrm{SUV}_{\max }$, maximum standardized uptake value; $\mathrm{SUV}_{\text {mean }}$, mean standardized uptake value; $\mathrm{N}$, number.

Age is also related to renal blood flow, glomerular filtration rate (GFR), and urea clearance, and the detailed mechanisms involved in these relationships may also facilitate the relationship between age and SUVs (26). It has been demonstrated that an age-related decline in GFR occurs during the human life span. After 30 years of age, a linear decline trend can be observed in GFR in healthy individuals. Moreover, previous studies have found that effective renal plasma flow was significantly lower in aging populations compared with the younger population (27). Similar clearance methods were also reported in infancy and childhood (28). The above physiological mechanisms are reminiscent of a potential explanation for age-related FDG uptake in the liver and blood pool.

Receptor, cytokine, and protein quality have been suggested to decrease with age, especially during the aging stages $(29,30)$, though it remains uncertain whether glucose transporter (GULT) numbers increase, decrease, or remain stable with age. To understand the mechanism of the relationship between SUVs and age, it is useful to first understand the effects of molecular substances on the age-related decline. ${ }^{18} \mathrm{~F}-\mathrm{FDG}$ is an analog of glucose that conjugates to glucose transport proteins to form FDG6-phosphate (FDG-6-P). This cannot be metabolized by glycolysis pathways and therefore accumulates in cells (31).
Glucose transmembrane transportation in the blood and hepatic tissue primarily depends on GLUT-1 and GLUT4 , respectively. The former is ubiquitous with basal-level glucose uptake and is found in erythrocytes and bloodtissue barriers, while the latter is the major hepatocyte isoform and is responsive to fed and fasting states $(32,33)$. Robust studies have shown that overexpression of GLUT1 and GLUT-3 results in a markedly enhanced demand for glucose in various solid tumors (34). Another study found that GLUT-1 plays a role in age-related insulin resistance, which may produce an indirect relationship between GLUT1 and age (35). Hotta et al. (36) reported a significant inverse correlation between age and mRNA levels of GLUT4 , which suggests that age is associated with GLUTs. As the most highly conserved and widely distributed glucose transporter in mammalian cells (37), GLUTs, which have been shown to change with age, may be a factor that can explain the association between age and SUVs.

Our study had some limitations, the first being that retrospective studies are inherently limited in nature. Secondly, due to incomplete height data collection, the influence of BMI on SUVs could not be analyzed. Thirdly, our study was based on a group of Chinese subjects with a specific scan vendor, incubation time, and reconstruction algorithm. It was also a single-center study. As such, our 
A

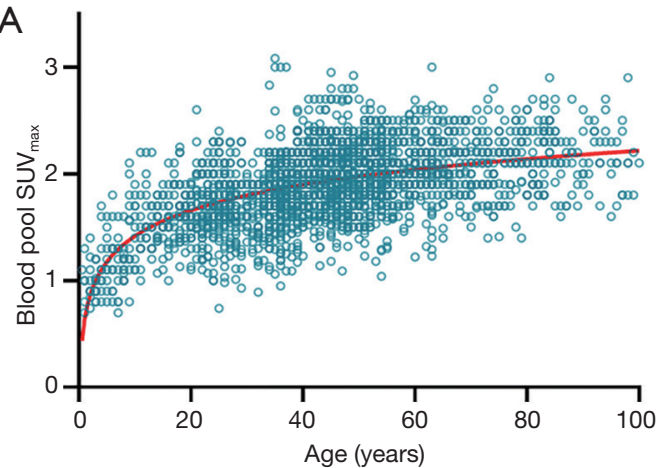

C

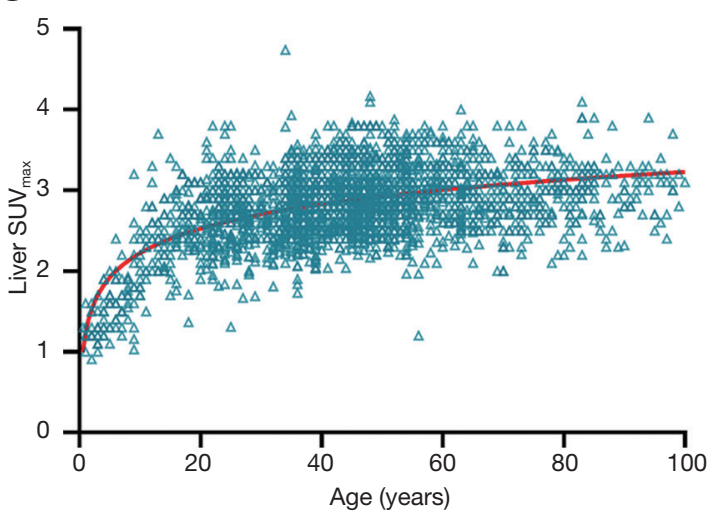

B

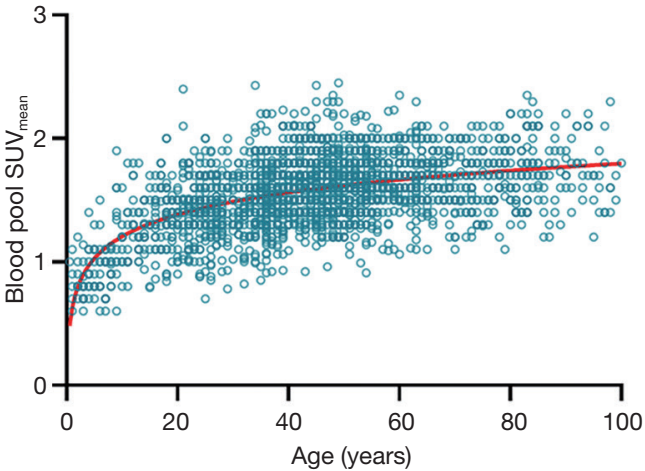

D

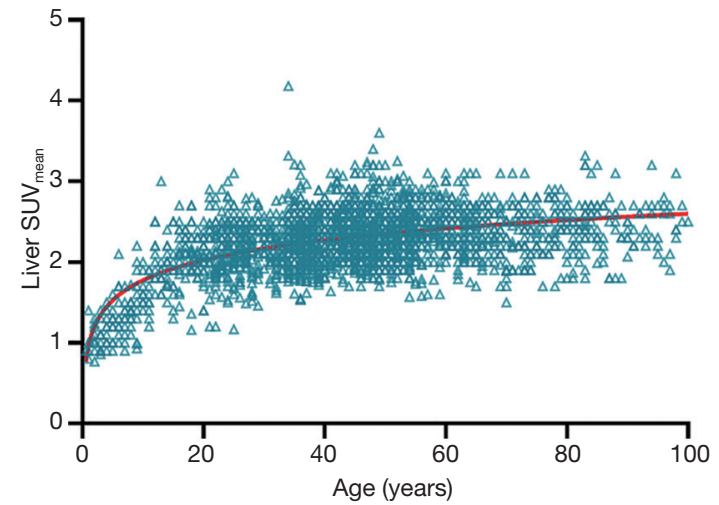

Figure $4 \mathrm{~A}$ scatter diagram of associations between age and SUVs. The SUVs of the blood pool and liver increased until the age of 20. The growth trend slowed and continued to the age of 100. (A) Age variation in blood pool $\mathrm{SUV}_{\text {max }}$ based on a large-scale population from 2009 to 2019. (B) Age variation in blood pool $S_{U_{\text {mean }}}$ based on a large-scale population from 2009 to 2019. (C) Age variation in liver $S U V_{\text {max }}$ based on a large-scale population from 2009 to 2019. (D) Age variation in liver SUV $_{\text {mean }}$ based on a large-scale population from 2009 to 2019. SUV, standardized uptake value.

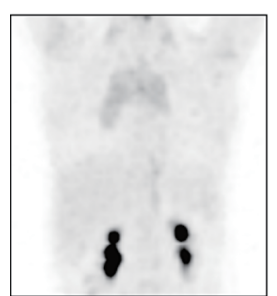

5 years

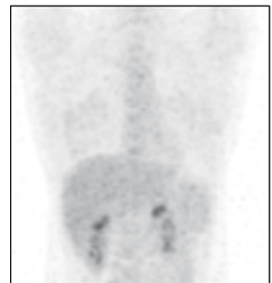

40 years

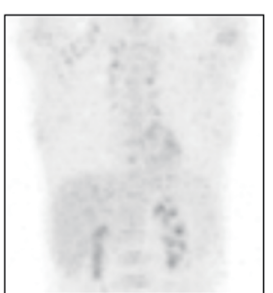

10 years

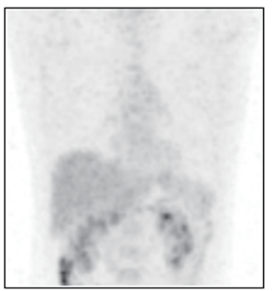

50 years

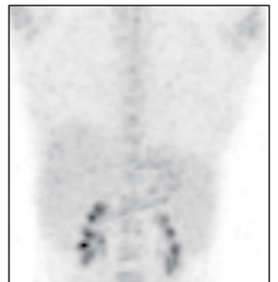

15 years

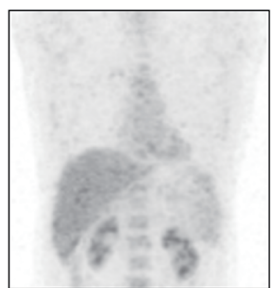

60 years

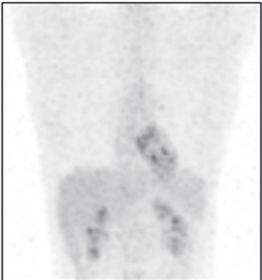

20 years

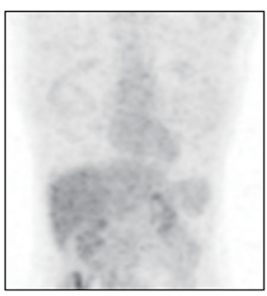

70 years

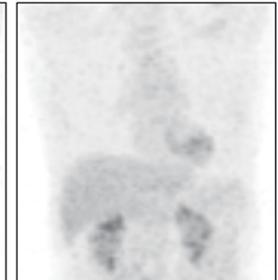

25 years

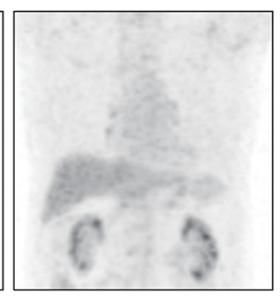

80 years

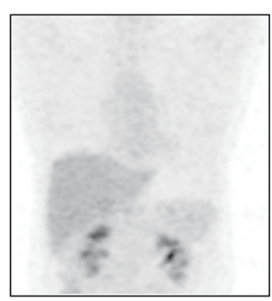

30 years

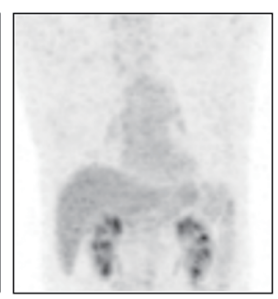

90 years

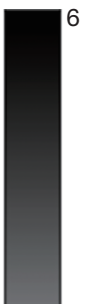

SUV

Figure 5 MIP images illustrating ${ }^{18} \mathrm{~F}$-FDG uptake in the liver and mediastinal blood pool across different ages $(5,10,15,20,25,30,40,50,60$, 70, 80, and 90 years). MIP, maximum intensity projection; ${ }^{18} \mathrm{~F}-\mathrm{FDG}$, fluorine-18-fluorodeoxyglucose. 
results must be verified in further studies that consider different populations to be deemed robust. Despite these limitations, the strength of our study cannot be ignored; its large sample size being evidence of its reliability and value. As this work was primarily focused on variations in SUVs with age, it lays the foundations for future studies to determine the clinical significance of age-related SUV changes. For example, if the liver or blood pool SUVs in a patient of a certain age were found to deviate from the normal range for that age as determined by PET/CT examination, the clinician should consider using blood pool and liver uptake as references for evaluating treatment response, especially when using Deauville scoring (5-PS) in the treatment of lymphoma or PET response criteria in solid tumor treatment.

\section{Conclusions}

Of all factors that influence the blood pool and liver SUVs, age was found to have the greatest impact. Physiological FDG uptake in the blood pool and liver showed significant variations across age groups. Blood pool and liver background SUVs increased with age and should be determined to fall within the specific reference ranges before oncologic whole-body PET/CT images are interpreted.

\section{Acknowledgments}

The authors thank Prof. Peng Huang from the Medical Research Center of Nanchang University for his statistical aid.

Funding: This study was supported by the National Natural Science Foundation of China (Grant Nos. 81971595 and 81771812), the Sichuan Province Science and Technology Support Program (Grant No. 2018SZ0391), and the 1.3.5 project for disciplines of excellence-Clinical Research Incubation Project, West China Hospital, Sichuan University (Grant No. 2020HXFH005).

\section{Footnote}

Reporting Checklist: The authors have completed the STROBE reporting checklist. Available at http://dx.doi. org/10.21037/qims-20-35

Conflicts of Interest: All authors have completed the ICMJE uniform disclosure form (available at http://dx.doi. org/10.21037/qims-20-35). The authors have no conflicts of interest to declare.

Ethical Statement: This study was approved by the ethics committee board of the West China Hospital of Sichuan University. This is a retrospective study, so no ethical approval or informed consent was obtained.

Open Access Statement: This is an Open Access article distributed in accordance with the Creative Commons Attribution-NonCommercial-NoDerivs 4.0 International License (CC BY-NC-ND 4.0), which permits the noncommercial replication and distribution of the article with the strict proviso that no changes or edits are made and the original work is properly cited (including links to both the formal publication through the relevant DOI and the license). See: https://creativecommons.org/licenses/by-nc-nd/4.0/.

\section{References}

1. Lin CY, Ding HJ, Lin CC, Chen CC, Sun SS, Kao CH. Impact of age on FDG uptake in the liver on PET scan. Clin Imaging 2010;34:348-50.

2. Cheng G, Alavi A, Lim E, Werner TJ, Del Bello CV, Akers SR. Dynamic changes of FDG uptake and clearance in normal tissues. Mol Imaging Biol 2013;15:345-52.

3. Paquet N, Albert A, Foidart J, Hustinx R. Within-patient variability of (18)F-FDG: standardized uptake values in normal tissues. J Nucl Med 2004;45:784-8.

4. Sprinz C, Zanon M, Altmayer S, Watte G, Irion K, Marchiori E, Hochhegger B. Effects of blood glucose level on 18F fluorodeoxyglucose (18F-FDG) uptake for PET/ CT in normal organs: an analysis on 5623 patients. Sci Rep 2018;8:2126.

5. Boktor RR, Walker G, Stacey R, Gledhill S, Pitman AG. Reference range for intrapatient variability in bloodpool and liver SUV for 18F-FDG PET. J Nucl Med 2013;54:677-82.

6. Boellaard R, Delgado-Bolton R, Oyen WJ, Giammarile F, Tatsch K, Eschner W, Verzijlbergen FJ, Barrington SF, Pike LC, Weber WA, Stroobants S, Delbeke D, Donohoe KJ, Holbrook S, Graham MM, Testanera G, Hoekstra OS, Zijlstra J, Visser E, Hoekstra CJ, Pruim J, Willemsen A, Arends B, Kotzerke J, Bockisch A, Beyer T, Chiti A, Krause BJ; European Association of Nuclear Medicine (EANM). FDG PET/CT: EANM procedure guidelines for tumour imaging: version 2.0. Eur J Nucl Med Mol Imaging 2015;42:328-54. 
7. Thie JA. Understanding the standardized uptake value, its methods, and implications for usage. J Nucl Med 2004;45:1431-4.

8. Roarke MC, Nguyen BD, Pockaj BA. Desmoplastic melanoma: true positive and false negative findings on F-18 FDG-PET/CT. Clin Nucl Med 2008;33:562-4.

9. Chen YK, Chen YL, Tsui CC, Wang SC, Cheng RH. The significance of alteration 2-[fluorine-18]fluoro-2-deoxy-(D)glucose uptake in the liver and skeletal muscles of patients with hyperthyroidism. Acad Radiol 2013;20:1218-23.

10. Sprinz C, Altmayer S, Zanon M, Watte G, Irion K, Marchiori E, Hochhegger B. Effects of blood glucose level on 18F-FDG uptake for PET/CT in normal organs: A systematic review. PLoS One 2018;13:e0193140.

11. Sarikaya I, Sarikaya A, Sharma P. Assessing effect of various blood glucose levels on (18)F-FDG activity in the brain, liver and blood pool. J Nucl Med Technol 2019;47:313-8.

12. Mahmud MH, Nordin AJ, Ahmad Saad FF, Azman AZ. Impacts of biological and procedural factors on semiquantification uptake value of liver in fluorine-18 fluorodeoxyglucose positron emission tomography/ computed tomography imaging. Quant Imaging Med Surg 2015;5:700-7.

13. Laffon E, Adhoute X, de Clermont H, Marthan R. Is liver SUV stable over time in (1)(8)F-FDG PET imaging? J Nucl Med Technol 2011;39:258-63.

14. Albano D, Bosio G, Pagani C, Re A, Tucci A, Giubbini R, Bertagna F. Prognostic role of baseline 18F-FDG PET/ CT metabolic parameters in Burkitt lymphoma. Eur J Nucl Med Mol Imaging 2019;46:87-96.

15. Chiaravalloti A, Danieli R, Abbatiello P, Di Pietro B, Travascio L, Cantonetti M, Guazzaroni M, Orlacchio A, Simonetti G, Schillaci O. Factors affecting intrapatient liver and mediastinal blood pool (1)(8)F-FDG standardized uptake value changes during ABVD chemotherapy in Hodgkin's lymphoma. Eur J Nucl Med Mol Imaging 2014;41:1123-32.

16. Schoen M, Braun T, Manava P, Ludwigs S, Lell M. Influence of scan time point and volume of intravenous contrast administration on blood-pool and liver SUVmax and SUVmean in [18F] FDG PET/CT. Nuklearmedizin 2018;57:50-5.

17. Laffon E, Milpied N, Marthan R. Measurement uncertainty of lesion and reference mediastinum standardized uptake value in lung cancer. Nucl Med Commun 2017;38:509-14.

18. Kamimura K, Nagamachi S, Wakamatsu H, Higashi R, Ogita M, Ueno S, Fujita S, Umemura Y, Fujimoto
T, Nakajo M. Associations between liver (18)F fluoro2-deoxy-D-glucose accumulation and various clinical parameters in a Japanese population: influence of the metabolic syndrome. Ann Nucl Med 2010;24:157-61.

19. Batallés SM, Villavicencio RL, Quaranta A, Burgos L, Trezzo S, Staffieri R, Pezzotto SM. Variations of the hepatic SUV in relation to the body mass index in whole body PET-CT studies. Rev Esp Med Nucl Imagen Mol 2013;32:26-32.

20. Kuruva M, Mittal BR, Abrar ML, Kashyap R, Bhattacharya A. Multivariate analysis of various factors affecting background liver and mediastinal standardized uptake values. Indian J Nucl Med 2012;27:20-3.

21. Wakabayashi H, Nishiyama Y, Ushiyama T, Maeba T, Maeta H. Evaluation of the effect of age on functioning hepatocyte mass and liver blood flow using liver scintigraphy in preoperative estimations for surgical patients: comparison with CT volumetry. J Surg Res 2002;106:246-53.

22. Watanabe T, Tanaka Y. Age-related alterations in the size of human hepatocytes. A study of mononuclear and binucleate cells. Virchows Arch B Cell Pathol Incl Mol Pathol 1982;39:9-20.

23. Meier JM, Alavi A, Iruvuri S, Alzeair S, Parker R, Houseni M, Hernandez-Pampaloni M, Mong A, Torigian DA. Assessment of age-related changes in abdominal organ structure and function with computed tomography and positron emission tomography. Semin Nucl Med 2007;37:154-72.

24. Geraghty EM, Boone JM, McGahan JP, Jain K. Normal organ volume assessment from abdominal CT. Abdom Imaging 2004;29:482-90.

25. Li Z, Wang Z. Aging Kidney and Aging-Related Disease. Adv Exp Med Biol 2018;1086:169-87.

26. Denic A, Glassock RJ, Rule AD. Structural and Functional Changes With the Aging Kidney. Adv Chronic Kidney Dis 2016;23:19-28.

27. Davies DF, Shock NW. Age changes in glomerular filtration rate, effective renal plasma flow, and tubular excretory capacity in adult males. J Clin Invest 1950;29:496-507.

28. Rodriguez-Soriano J, Vallo A, Castillo G, Oliveros R. Renal handling of water and sodium in infancy and childhood: a study using clearance methods during hypotonic saline diuresis. Kidney Int 1981;20:700-4.

29. Murray TM, Desai KS, Cinader B. Age-related changes in hepatic and splenic insulin receptors and serum insulin and glucose levels in inbred mice. Metabolism 1993;42:140-4. 
30. Kohno A, Cinader B, Seeman P. Age-related changes in beta-adrenoceptors of lymphocytes. Immunol Lett 1986;13:79-82.

31. Kostakoglu L, Agress H Jr, Goldsmith SJ. Clinical role of FDG PET in evaluation of cancer patients. Radiographics 2003;23:315-40; quiz 533.

32. Deng D, Sun P, Yan C, Ke M, Jiang X, Xiong L, Ren W, Hirata K, Yamamoto M, Fan S, Yan N. Molecular basis of ligand recognition and transport by glucose transporters, Nature 2015;526:391-6.

33. Dick AP, Harik SI, Klip A, Walker DM. Identification and characterization of the glucose transporter of the bloodbrain barrier by cytochalasin B binding and immunological reactivity. Proc Natl Acad Sci U S A 1984;81:7233-7.

34. Macheda ML, Rogers S, Best JD. Molecular and cellular regulation of glucose transporter (GLUT) proteins in cancer. J Cell Physiol 2005;202:654-62.

35. Mohamad M, Mitchell SJ, Wu LE, White MY, Cordwell

Cite this article as: Cao Y, Zhou K, Diao W, Long X, Tian F, Su M, Jia Z. Age-related changes of standardized uptake values in the blood pool and liver: a decade-long retrospective study of the outcomes of 2,526 subjects. Quant Imaging Med Surg 2021;11(1):95-106. doi: 10.21037/qims-20-35
SJ, Mach J, Solon-Biet SM, Boyer D, Nines D, Das A, Catherine Li SY, Warren A, Hilmer SN, Fraser R, Sinclair DA, Simpson SJ, de Cabo R, Le Couteur DG, Cogger VC. Ultrastructure of the liver microcirculation influences hepatic and systemic insulin activity and provides a mechanism for age-related insulin resistance. Aging Cell 2016;15:706-15.

36. Hotta K, Bodkin NL, Gustafson TA, Yoshioka S, Ortmeyer HK, Hansen BC. Age-related adipose tissue mRNA expression of ADD1/SREBP1, PPARgamma, lipoprotein lipase, and GLUT4 glucose transporter in rhesus monkeys. J Gerontol A Biol Sci Med Sci 1999;54:B183-8.

37. Cho SJ, Moon JS, Lee CM, Choi AM, Stout-Delgado HW. Glucose Transporter 1-Dependent Glycolysis Is Increased during Aging-Related Lung Fibrosis, and Phloretin Inhibits Lung Fibrosis. Am J Respir Cell Mol Biol 2017;56:521-31. 
Supplementary

Table S1 Correlations between SUVs with related factors (>18 years)

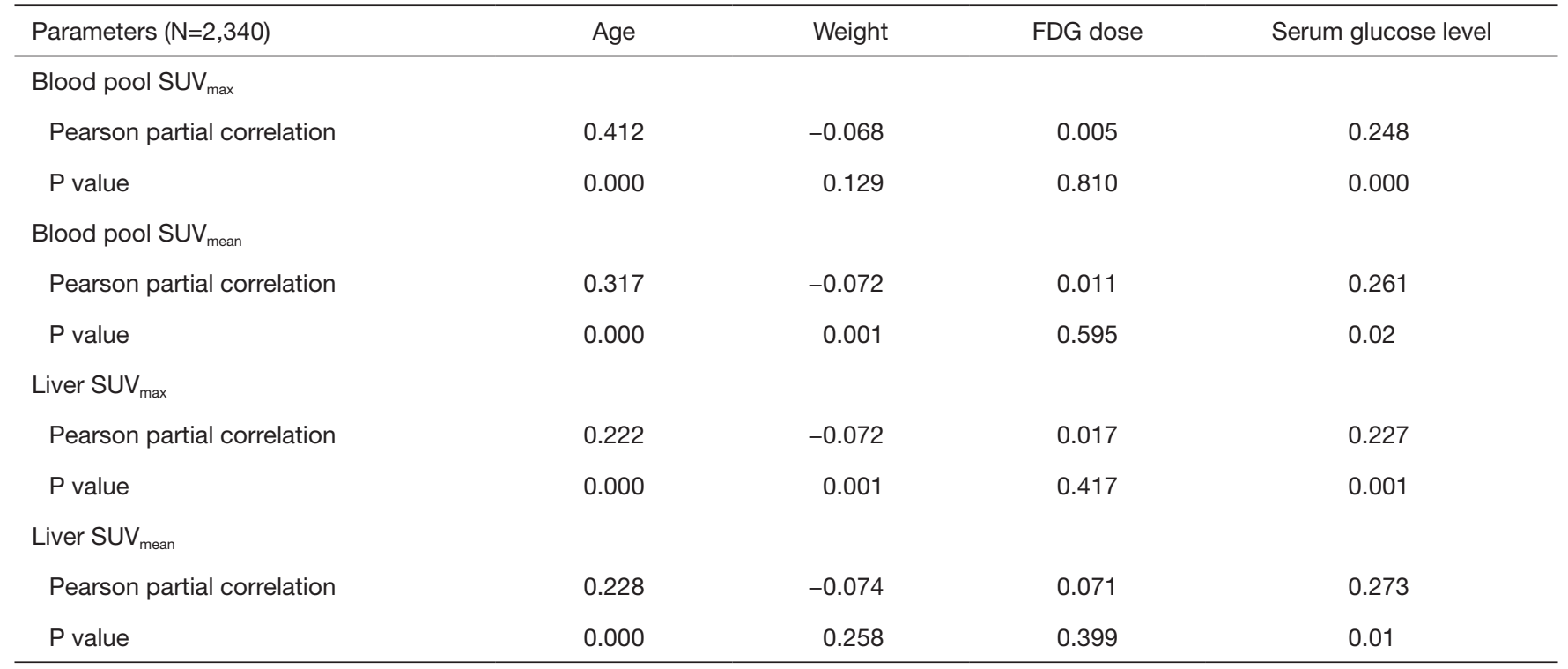

${ }^{18}$ F-FDG, fluorine-18-fluorodeoxyglucose; $\mathrm{SUV}_{\max }$, maximum standardized uptake value; SUV $_{\text {mean, }}$ mean standardized uptake value; $\mathrm{N}$, number.

Table S2 Impact of biological and procedural related factors on SUVs of blood pool and liver in multivariate regression analysis ( $>18$ years)

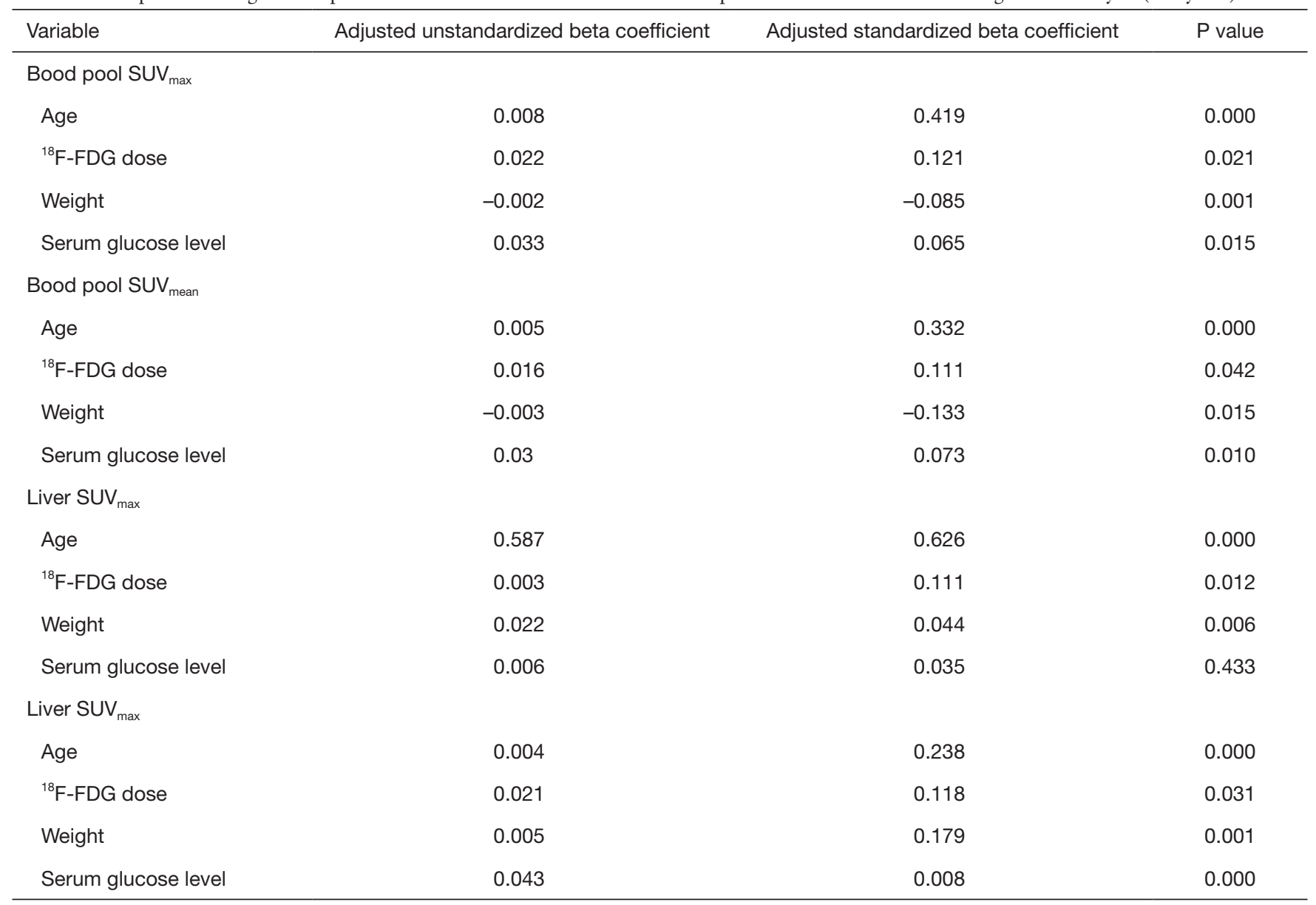

${ }^{18}$ F-FDG, fluorine-18-fluorodeoxyglucose; $\mathrm{SUV}_{\max }$, maximum standardized uptake value; $\mathrm{SUV}_{\text {mean }}$, mean standardized uptake value; $\mathrm{N}$, number. 